\title{
Research article \\ Evaluation of efficacy of different tooth paste formulations in reducing the oral microbial load - An in vivo study

\author{
Priyal G. ${ }^{1}$, Maji Jose ${ }^{2}$, Shruti Nayak ${ }^{3}$, Vidya Pai ${ }^{4}$, Sudeendra Prabhu ${ }^{5}$ \\ ${ }^{1}$ Senior Lecturer, AJ Shetty Institute of Dental Sciences, Mangalore, Karnataka, India \\ ${ }^{2}$ Professor and Vice Principal, ${ }^{3}$ Reader, Department of Oral Pathology, ${ }^{5}$ Professor of Oral Pathology and In-charge Head, \\ Centre for Forensic Odontology, Yenepoya Dental College, ${ }^{4}$ Professor, Department of Microbiology, Yenepoya Medical \\ College, Yenepoya Deemed to be University, Mangalore, Karnataka, India
}

(Received: January $2021 \quad$ Revised: June $2021 \quad$ Accepted: July 2021)

Corresponding author: Sudeendra Prabhu. Email: drsudi2014@gmail.com

\begin{abstract}
Introduction and Aim: Toothpastes are the most common preventive means in oral health care. Many commercially available dentifrices claim to have antimicrobial properties, but little research has been conducted to investigate these claims. Therefore, this study was conducted to evaluate the efficacy of different toothpaste formulations in reducing the oral microbial load.

Methodology: A total of six types of tooth pastes- 4 herbal and 2 non-herbal- were assigned to a group of 20 subjects each, who used it for a period of six weeks. During the course of the study, at the end of $1^{\text {st }}, 3^{\text {rd }}$ and $6^{\text {th }}$ week, subgingival plaque samples collected were subjected to microbial analysis.

Results: Microbial analysis identified colonies of organisms such as S. mitis, S. mutans, S. salivarius, Peptostreptococcus species, Prevotella species and Lactobacillus species. Colony forming units (cfu) calculated indicated a reduction in microbial load from $1^{\text {st }}$ to $6^{\text {th }}$ week of use of different tooth paste formulations. However statistical analysis of the results did not show significant differences between the groups ( $p>0.005)$.

Conclusion: The selected tooth paste formulations were effective in controlling the microbial load and therefore contributing to maintain good oral hygiene. However, practicing appropriate oral hygiene measures \& brushing technique is of utmost importance in maintaining good oral health than the effectiveness of various ingredients in the toothpastes used.
\end{abstract}

Keywords: Dentifrices; tooth pastes; herbal; non herbal; plaque; microflora; antimicrobial efficacy.

\section{INTRODUCTION}

$\mathrm{H}$ ealth is a universal human need for all cultural groups, and it has been established beyond doubt that general health cannot be attained or maintained without oral health (1). A healthy mouth has dynamic mutualistic microbial interactions within it and, the ambient temperature with the regular presence of moisture and nutrients further makes the mouth a paradise in which bacteria thrive and multiply (2). In general, when we see, the oral cavity is sterile at birth, but as time proceeds, it becomes colonized with micro-organisms from the environment, mostly from the mother in the first feeding. The most predominant microbe to inhabit in the oral cavity in the initially is Streptococcus salivarius. Till the appearance of teeth, Streptococcus salivarius makes up $98 \%$ of the total oral flora, this happens around 6 months after birth. These bacteria require a non-desquamating (non-epithelial) surface to colonize, and they persist as long as the teeth remain in the oral cavity.

One of the most common effort of modern dentistry is the importance of dental plaque control to improve oral health (3) and reduce the incidence of these clinical conditions (4). Personal oral hygiene measures are generally considered the mainstay of oral disease prevention, and dental professionals include oral hygiene instructions for patients, especially for those who present with high levels of dental plaque (5).

It is well understood that more than 2000 years back, we have followed to carry out the use of dentifrices as a tooth cleaning practice and toothpicks and brushes remains to be as even older practice. Today's dentifrices follow many of the same principles that were developed centuries ago, the difference being that modern products have a higher degree of compositional sophistication and improvements in esthetics and therapeutic value. The ability to get rid of pathogenic microorganisms and diminish the risk of infection in the mouth is determined by the efficiency and effectiveness of any toothpaste.

Use of herbal or Ayurvedic medicines for overall health in general and oral health is an integral part of Indian tradition. Many ayurvedic toothpastes are available in the market claiming to have good antimicrobial properties. However very little research has been conducted to investigate the effectiveness of herbal toothpaste compared to non-herbal toothpastes in reducing the oral microbial load. Hence, an attempt has been made in the present study to assess and 
compare the in-vivo antimicrobial potential of two herbal and two non-herbal toothpastes (6).

\section{MATERIALS AND METHODS}

Participants consisted of 120 healthy volunteers of 18-20 years who were randomly selected from students of Dental College. Selected participants were divided into six groups of 20 participants each. At the commencement of the study, a thorough oral prophylaxis was done and Gingival index of Loe and Sillness and Plaque index of Sillness and Loe were recorded for every participant to ensure the bacterial load is minimal. To standardize the brushing technique, oral hygiene instructions were given. To ensure and make sure that all participants follow the same oral hygiene procedure, they were provided with standard toothbrush which they used during the study period and were instructed not to use any other oral hygiene measures, other than instructed.

While selecting the individuals the inclusion and exclusion criteria were strictly followed. Subjects with gingival index score of 0.1-1 were only included for the study that ranged from mild inflammation to no inflammation and sound gingival status. Subjects who were undergoing orthodontic treatment, with a habit of continuous usage of chewing gum / use of mouth rinses were not included.

Six types of toothpastes were selected for this study: four different herbal toothpaste and two non herbal with distinctive active ingredients, namely Neem, Meswak, Babool, Mango Leaves, Triclosan \& Sodium Triclosan, and Sodium and Monofluoro Phosphate. The toothpastes were coded as 001, 002, 003, 004, 005, 006 and assigned to specific group of participants who were instructed to brush twice daily with the given tooth paste for a period of 6 weeks.
During this period of study, subgingival plaque samples were collected from each volunteer at three different instances i.e., end of $1^{\text {st }}, 3^{\text {rd, }}$ and $6^{\text {th }}$ week. The plaque samples were collected from mesolingual gingival sulcus of mandibular left first molar-under strict aseptic conditions using a sterile ISO 45 paper point.

Samples collected were transferred into a bottle containing Robertson's cooked meat medium (RCM) broth and transported to Department of Microbiology where microbial analysis was carried out.

\section{Microbial analysis}

10 micro liters of inoculated RCM broth was sub-cultured on Mitis Salivarius agar and McConkey agar immediately. RCM broth was incubated at $37^{\circ} \mathrm{C}$ for $24 \mathrm{hrs} .10$ micro liters of incubated broth is sub-cultured on anaerobic blood agar on which a disc of metronidazole was applied in the primary streak and the plate was then incubated at $37^{\circ} \mathrm{C}$ for $72 \mathrm{hrs}$ in anaerobic chamber. The colonies on Mitis Salivarius agar/ McConkey agar and anaerobic blood agar were inspected and identified using the colony morphology, gram staining, catalase test, metronidazole sensitivity, aerotolerance test and pigment production as per standard microbiological methods. The number of colonies of each type of bacteria was counted using a colony counter and colony forming units (cfu) per millilitre was calculated by multiplying with conversion factor. The colonies of following organisms were identified- Streptococcus mitis, Streptococcus mutans, Streptococcus salivarius, Peptostreptococcus species, Prevotella species, Bacteroides species and Gram-negative aerobic bacilli (Fig. 1).

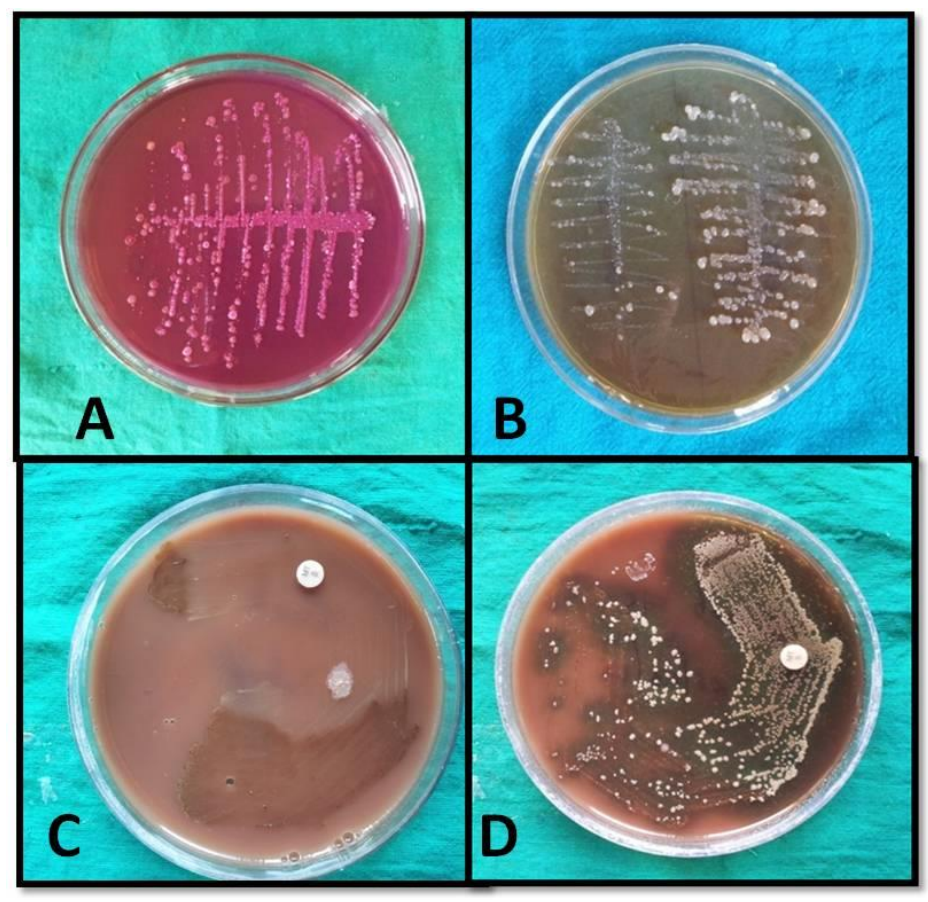

Figure 1: Mc Conkey agar plate (A), Msa plate showing (B), Anaerobic blood agar plate with a metronidazole disc showing growth of black coloured colonies (C), Blood agar plate showing tiny transpare (D). 


\section{RESULTS}

The plaque samples collected were analyzed for microbial load. The antimicrobial effect of various tooth paste formulations were tested against specific micro-organisms by recording colony forming units (cfu) per milliliter in cultured plaque samples, collected during specific intervals of the study. The number of colonies < $10 \mathrm{cfu}$ was considered as negative and colonies >10 cfu was considered positive. The results were then subjected to statistical analysis.

Plaque samples from $85 \%$ of participants who used tooth paste 001, presented with Streptococcus mitis at $1^{\text {st }}$ week of study which dropped down to $4 \%$ by $6^{\text {th }}$ week. Similarly, tooth paste 005 and 006 also showed a reduction from $80-50 \%$ and $65-45 \%$ respectively. However, 90-95\% participants who used pastes 002, $003 \& 004$ had Streptococcus mitis in the plaque sample and remained unchanged from $1^{\text {st }}$ to $6^{\text {th }}$ week. When these findings were statistically analyzed, results with respect to tooth paste 001 was highly
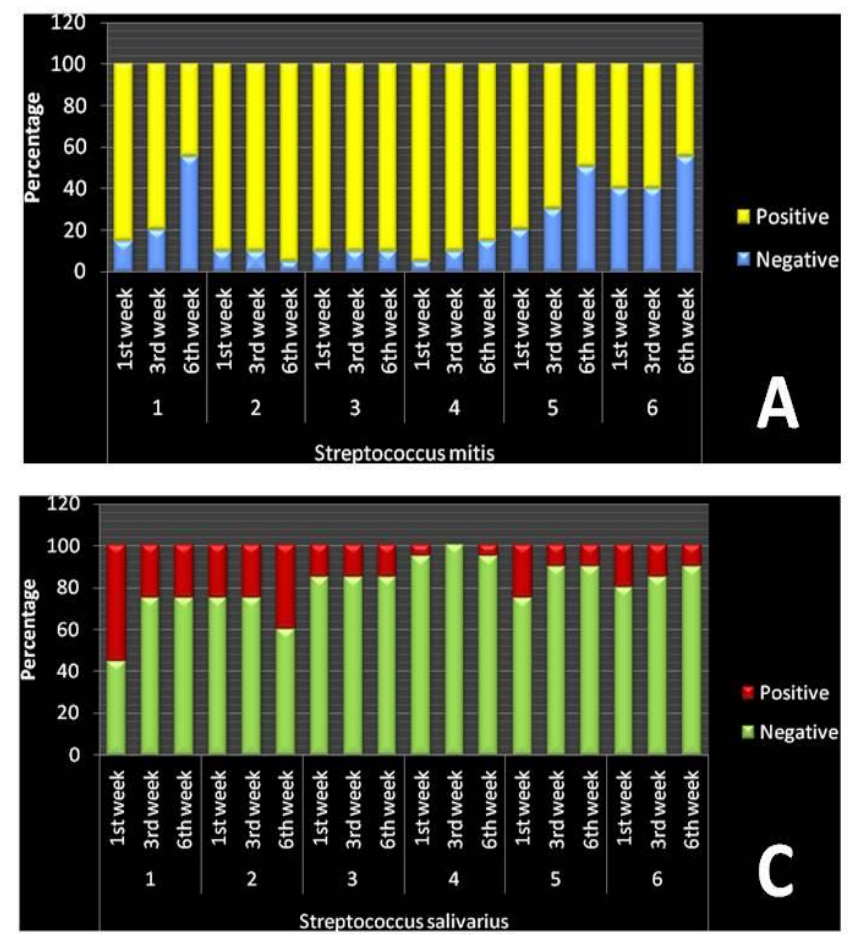

significant ( $\mathrm{p}$ value 0.008 ), tooth paste 005 was significant ( $p$ value 0.046) while 006 was not significant (p value 0.083) (Table 1, graph 1). When the efficacy of different toothpastes was compared between each other, it is noted that tooth paste samples 001, 005 and 006 were more effective against $S$. mitis compared to other three.

When Streptococcus mutans were checked in plaque samples, after use of tooth paste 001, $75 \%$ of participants showed the growth at the end of $1^{\text {st }}$ week, which decreased to $65 \%$ (not statistically significant, $p$ value 0.317). All other toothpastes showed a significant reduction in $S$. mutans count within $1^{\text {st }}$ week of use with only 40-50\% participants showing positivity. Tooth paste samples 002, 003, $005 \& 006$ did not show further reduction while sample 004 showed significant reduction from $40-10 \%$ by the end of $6^{\text {th }}$ week ( $\mathrm{p}$ value 0.034 , Table 1 , Graph 1 ). Tooth paste sample 004 was found to be more effective when compared to other samples against $S$. mutans.
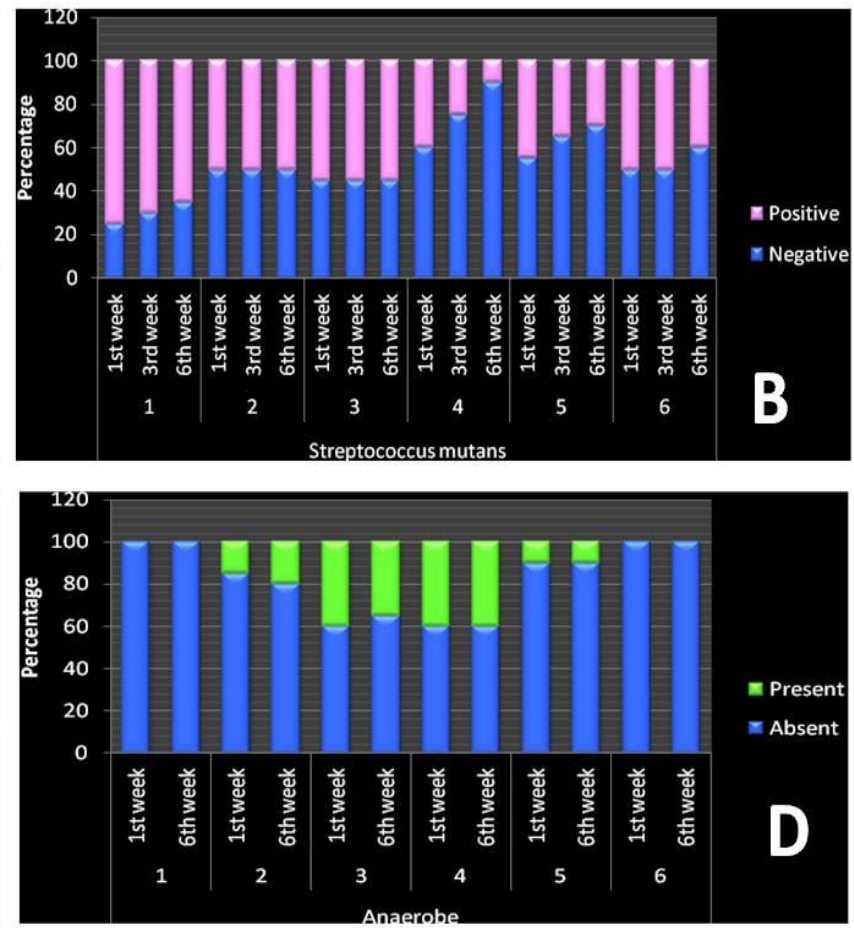

GRAPH 1: Quantitative changes in Streptococcus mitis (A), Quantitative changes in Streptococcus mutans (B), Quantitative changes in Streptococcus salivarius $(C)$, Quantitative changes in Anaerobic organisms.

Number of participants exhibiting Streptococcus salivarius in the plaque samples, $1^{\text {st }}$ week after use of different toothpaste were $55 \%, 25 \%, 15 \%, 5 \%, 25 \%$ and $20 \%$ with tooth paste samples $001,002,003,004,005$, and 006 respectively. No reduction was noted except for tooth paste sample 001 which showed a statistically significant reduction from $55 \%$ to $25 \%$ by the end of $6^{\text {th }}$ week of use ( $\mathrm{p}$ value 0.046 , Table 1 , Graph 1). No significant difference was noted between the toothpastes, in their effect against $S$. salivarius.
When the cultured plaque samples were examined for anaerobic organisms, nearly $100 \%$ of participants in group $001 \& 006$, showed absence of significant colony forming units (cfu) and this finding remained consistent from $1^{\text {st }}$ to $6^{\text {th }}$ week. All other toothpaste samples also showed their effectiveness against these groups of organisms with less percentage of participants presenting with positivity even at the end of $1^{\text {st }}$ week with minor reduction by end of $6^{\text {th }}$ week. When the efficacy was evaluated between different tooth paste samples, 001, 002, 005 \& 006 were comparable and was significantly better than 003 and 004 (Table 2). 
Table 1: Comparison among the groups, Post hoc analysis by Mannwhitney test

\begin{tabular}{|ll|r|r|r|r|r|r|}
\hline & & \multicolumn{2}{|c|}{ 3rd week - 1st week } & \multicolumn{2}{c|}{ 6th week - 1st week } & \multicolumn{2}{c|}{ 6th week - 3rd week } \\
\cline { 2 - 8 } organism & Group & $\begin{array}{c}\text { Z test } p \\
\text { value }\end{array}$ & & $\begin{array}{c}\text { Z test } p \\
\text { value }\end{array}$ & & $\begin{array}{c}\text { Z test } p \\
\text { value }\end{array}$ & \\
\hline Streptococcus mitis & 001 & .317 & & .005 & sig & .008 & HS \\
& 002 & 1.000 & & .317 & & .317 & \\
& 003 & 1.000 & & 1.000 & & 1.000 & \\
& 004 & .564 & & .317 & & .317 & \\
& 005 & .157 & & .014 & sig & .046 & sig \\
& 006 & 1.000 & & .083 & & .083 & \\
\hline Streptococcus mutans & 001 & .317 & & .157 & & .317 & \\
& 002 & 1.000 & & 1.000 & & 1.000 & \\
& 003 & 1.000 & & 1.000 & & 1.000 & \\
& 004 & .317 & & .034 & sig & .083 & \\
& 005 & .157 & & .083 & & .317 & \\
& 006 & 1.000 & & .157 & & .157 & \\
\hline Streptococcus salivarius & 001 & .014 & sig & .046 & sig & 1.000 & \\
& 002 & 1.000 & & .180 & & .180 & \\
& 003 & 1.000 & & 1.000 & & 1.000 & \\
& 004 & .317 & & 1.000 & & .317 & \\
& 005 & .083 & & .083 & & 1.000 & \\
& 006 & .317 & & .157 & & .317 & \\
\hline
\end{tabular}

Table 2: Comparison among the groups for presence of Anaerobic organisms

\begin{tabular}{|c|c|c|c|c|c|c|c|c|}
\hline \multirow{2}{*}{ organism } & \multirow[b]{2}{*}{ Group } & & \multicolumn{2}{|c|}{ Absent } & \multicolumn{2}{|c|}{ Present } & \multicolumn{2}{|c|}{ Total } \\
\hline & & & Freq & $\%$ & Freq & $\%$ & Freq & $\%$ \\
\hline \multirow[t]{12}{*}{ Anaerobe } & 001 & 1st week & 20 & $100.0 \%$ & & & 20 & $100.0 \%$ \\
\hline & & 6th week & 20 & $100.0 \%$ & & & 20 & $100.0 \%$ \\
\hline & 002 & 1st week & 17 & $85.0 \%$ & 3 & $15.0 \%$ & 20 & $100.0 \%$ \\
\hline & & 6th week & 16 & $80.0 \%$ & 4 & $20.0 \%$ & 20 & $100.0 \%$ \\
\hline & 003 & 1st week & 12 & $60.0 \%$ & 8 & $40.0 \%$ & 20 & $100.0 \%$ \\
\hline & & 6th week & 13 & $65.0 \%$ & 7 & $35.0 \%$ & 20 & $100.0 \%$ \\
\hline & 004 & 1st week & 12 & $60.0 \%$ & 8 & $40.0 \%$ & 20 & $100.0 \%$ \\
\hline & & 6th week & 12 & $60.0 \%$ & 8 & $40.0 \%$ & 20 & $100.0 \%$ \\
\hline & 005 & 1st week & 18 & $90.0 \%$ & 2 & $10.0 \%$ & 20 & $100.0 \%$ \\
\hline & & 6 th week & 18 & $90.0 \%$ & 2 & $10.0 \%$ & 20 & $100.0 \%$ \\
\hline & 006 & 1st week & 20 & $100.0 \%$ & & & 20 & $100.0 \%$ \\
\hline & & 6th week & 20 & $100.0 \%$ & & & 20 & $100.0 \%$ \\
\hline
\end{tabular}

\section{DISCUSSION}

Tooth brushing with a dentifrice is the most common method of oral hygiene practices and therefore, toothpaste is a potentially ideal vehicle for agents promoting oral health benefits. Consumers are exposed to advertising campaigns that highlight the benefits of a variety of over-the-counter herbal and non-herbal toothpastes. As a result, consumers are in a dilemma about the use of these products. Hence the present study was undertaken to assess the in-vivo antimicrobial efficacy of selected commercially available herbal and non-herbal toothpastes. The herbal and non-herbal toothpastes tested in this study contained several different constituents with putative anti-inflammatory and anti-bacterial properties, which theoretically could be useful in controlling the oral microbial population.
The antimicrobial effect of selected tooth paste samples were analyzed by quantifying the microbial load in the plaque samples collected at different time intervals after the use of specific tooth paste. The antimicrobial efficacy of six different tooth paste formulations were tested against aerobic micro-organisms, particularly cariogenic organisms such as Streptococcus mutans, Streptococcus mitis \& Streptococcus Salivarius, as well as anaerobic organisms like, Pepto-streptococcus species, Prevotella species \& Lactobacillus species. The number of colonies of each type of bacteria was counted using a colony counter and was interpreted as positive when the number of colonies were $>10 \mathrm{cfu}$ and negative when $<10 \mathrm{cfu}$. In the present study, we have used four herbal toothpastes containing extracts of neem, miswak, babool and mango leaves as the major ingredients. All these herbal materials are 
proven to have antimicrobial effects against a number of aerobic and anaerobic micro-organisms, in in-vivo as well as few in-vitro studies (7).

In the present study, all selected toothpaste samples showed reduction in microbial load in plaque sample collected at different time intervals during the study. With respect to $S$. mutans, tooth paste sample 004 i.e., herbal toothpaste containing Babool extract as major ingredient was found to be more effective. However other samples also exhibited inhibitory effect within short duration of use. Tooth paste samples, 001 containing neem extract as major ingredient, 005 containing triclosan and sodium monofluorophosphate and 006 containing mango leaf extracts, were noted to be more effective against $S$. mitis than other samples. Significant number of participants showed considerable reduction in $S$. salivarius in their plaque samples even with one week of use of different selected tooth paste samples. The efficacy in inhibiting anaerobic organism was superior with different herbal tooth pastes $001,002, \& 006$ and 005, containing triclosan and sodium monofluorophosphate.

The major biologically active substances in neem are limonoids, azadirone, nimbin, salanin, alkaloids, flavonoids, triterpenoids, phenolic compounds, carotenoids, steroids, and ketones.

Oil from the leaves, seed and bark possesses a wide spectrum of antibacterial action against Gram-negative and Gram-positive micro-organisms. Study carried out by Patil et al., (8). on neem containing herbal toothpaste proved neem extracts in toothpaste, can impart antimicrobial effects comparable to fluoridated toothpaste. Therefore, the evidence in the literature supports our observation of the effect of neem extract on the oral microflora.

Similarly, the major biologically active components in miswak are trimethylamine, salvadorine, chlorides, silica, sulfur, vitamin $\mathrm{C}$ and small quantities of tannins, saponins, flavonoids and sterols (9). Various in-vitro studies confirmed efficient antimicrobial activities of miswak towards Staphylococcus aureus, Streptococcus mutans, Lactobacillus acidophilus, E. coli and Pseudomonas aeruginosa (10-12). This effectiveness is also established in in-vivo study conducted by Gupta et al., (12) who reported the anti-plaque efficacy of miswak containing dentifrice, comparable with the conventional fluoridated dentifrice. The participants of our study, who used tooth paste which contained miswak as major ingredient showed improvement in oral health and reduction in microbial load and thus our results are consistent with earlier report.

Babool, which is botanically known as Acacia Arabica, is also rich in biologically active components such as flavonoids, sterols, triterpenoids, alkaloids and phenolic substances imparting it significant antimicrobial properties (13). Few in-vitro studies have shown that dentifrices containing babool as the active component appear to be equally effective as that of the conventional toothpastes on the micro-organisms, when used in combination with neem $(14,15)$. These evidences are in par with our findings. The main biologically active components in mango leaf extract are phenolic constituents, triterpenes, flavonoids, phytosterols, polyphenols. Among these components, Mangiferin is of particular significance because of numerous therapeutic effects including antitumor, antiviral, antidiabetic, anti-inflammatory and potent antioxidant properties (16). Effectiveness of Mangiferin against Streptococci and Lactobacillus acidophilus was confirmed in previous research (17-20).

The herbal extracts have numerous beneficial medicinal effects which includes, antibacterial activity naturally results from the secondary products present in the plant even though, it is not attributed usually to a single compound but a combination of metabolites. How the active components of the plant materials contribute to the antibacterial activity, the exact mechanism is not known. Among all, one of the suggested mechanisms is hydrophobic activity, which enables them to partition the lipids of the bacterial cell membrane and mitochondria, disturbing the cell structure and rendering them more permeability. It is observed that bacterial cell death can occur from extensive leakage from bacterial cells or the exit of critical molecules and ions.

In the present research work, we have also analyzed the antimicrobial effects of two non herbal toothpastes in addition to the herbal toothpastes. Tooth paste sample 003 contained triclosan as the active ingredient and tooth paste sample 005 had sodium monofluorophosphate as additional component with triclosan. Triclosan has a broad spectrum of antimicrobial activity against yeasts and oral bacteria. Triclosan acts as a biocide at high concentrations, with multiple cytoplasmic and membrane targets However, at the lower concentrations seen in commercial products, triclosan appears bacteriostatic, and it is seen to target bacteria primarily by inhibiting fatty acid synthesis. Triclosan is combined either with a co-polymer or with another compatible antimicrobial agent, zinc citrate, to enhance its clinical efficacy. The copolymer acts to increase the oral retention of triclosan, and result in further reduction in salivary bacterial counts.

Tooth paste sample 005 containing sodium monofluorophosphate and triclosan as the active ingredients has been proved earlier for its anti-cariogenic and antimicrobial effect. Fluoride in various forms is the most popular active ingredient in tooth paste to prevent cavities. The mechanism of action could be related to the ability of fluoride to absorb to the surface of the apatite crystals, when 
present in low sustained concentrations (sub-ppm range) in the oral fluids during an acidic challenge, inhibiting demineralization. Traces of fluoride in solution will make it highly supersaturated with respect to fluorhydroxyapatite, When the $\mathrm{pH}$ is re-established which will speed up the process of remineralization. The mineral formed under the nucleating action of the partially dissolved minerals will then preferentially include fluoride and exclude carbonate, rendering the enamel more resistant to future acidic challenges. When the $\mathrm{pH}$ is re-established, traces of fluoride in solution will make it highly supersaturated with respect to fluorhydroxyapatite, which will speed up the process of remineralization. The mineral formed under the nucleating action of the partially dissolved minerals will then preferentially include fluoride and exclude carbonate, rendering the enamel more resistant to future acidic challenges (21). Sodium fluoride (NaF) is the most common source of fluoride, but stannous fluoride $\left(\mathrm{SnF}_{2}\right)$, olaflur and sodium monofluoro phosphate $\left(\mathrm{Na}_{2} \mathrm{PO}_{3} \mathrm{~F}\right)$ are also used. It has been proven that fluorides are more effective than sodium fluoride in reducing the incidence of dental caries (22) and controlling gingivitis (23).

Many new dentifrices and other oral care products are getting introduced to the market claiming contributing to better oral health. Because of increasing popularity and acceptance of herbal products among the public, various commercial companies have come up with different herbal toothpastes incorporating different herbal components as active ingredients. Thus, the Indian market is flooded with numerous toothpaste brands of wide range of price, ultimately dragging common people and even dental professionals into a state of confusion while selecting and recommending the appropriate toothpaste. Therefore, choosing appropriate tooth paste for a family has become a herculean task for many. In our study we noted that, all selected toothpastes; herbal and non-herbal, exhibited reduction in microbial load and resulted in improvement in oral health status, without significant differences between them. To what extent this effect is available in the oral cavity to reduce the microbial load is debatable. However, we would like to state that, practicing proper oral hygiene measures and brushing technique is of utmost importance in maintaining good oral health than the effectiveness of various ingredients in the toothpastes used. Our study highlights the need of strategies to improve the awareness of public on importance of oral hygiene measures and proper brushing technique. Owing to the constraints, pertaining to available time, cost, and manpower, practically it was difficult to include all the toothpastes existing in the market. Further studies using more toothpaste samples and detailed qualitative and quantitative analysis of plaque microbial flora using sophisticated microbial detection techniques is essential to substantiate our observation.

\section{CONCLUSION}

Since the toothpastes remain in the oral cavity only for a transient period and of the active ingredients get immediately diluted in saliva during brushing, the relevance variation in active component of the tooth paste in contributing to good oral health is debatable. Therefore, we would like to conclude that, practicing appropriate oral hygiene measures and brushing technique is of utmost importance in maintaining good oral health than the ingredients of the toothpastes used.

More research is needed to validate the effectiveness claims of various tooth paste formulations available in the market and that this study provides practitioners with an insight into the claims of both herbal and non-herbal dentifrices antimicrobial effects.

\section{CONFLICT OF INTEREST}

Authors declare no conflict of interest.

\section{REFERENCES}

1. Oral health- Global Health Testimony for the 2005 Global Health Summit Philadelphia, Pennsylvania June 5, 2005.

2. Global Review on Oral Health in Ageing Societies WHO Kobe Centre for Health Development Ageing and Health Technical Report. 2002; (3).

3. Hatti, S., Ravindra, S., Sathpathy, A., Kulkarni, R. D., Parande, M. V. Biofilm inhibition and antimicrobial activity of a dentifrice containing salivary substitutes. Int J Dent Hygiene 2007; 5: 218-224.

4. Harris, N., Franklin, G. G. Primary preventive dentistry. 6th edition Pearson prentice hall upper saddle River, New Jersey.

5. Todar, K. The bacterial flora of humans. Todar's online textbook of bacteriology 2007.

6. Abdel Rahman, H. F., Skaug, N., Francis, G. W. In vitro antimicrobial effects of crude miswak extracts on oral pathogens. Saudi Dental Journal. 2002; 1(14).

7. Almas, K., AL-Zeid, Z. The immediate antimicrobial effect of a tooth brush and miswak on cariogenic bacteria: a clinical study. J Cotemp Dent Pract. 2004; 5: 105-114.

8. Patil, S., Venkataraghavan, K., Anantharaj, A., Patil, S. Comparison of two commercially available toothpastes on the salivary streptococcus mutans count in urban preschool children -an in vivo study. International dentistry SA; 12(4): 72-78.

9. Jose, S., Beegum, G. R. J. In vitro susceptibility of viridans streptococci to leaf extracts of Mangifera indica. Indian Journal of Microbiology. 2007; 47: 160-163.

10. Prashant, G. M., Chandu, G. N., Murulikrishna, K. S., Shafiulla, M. D. The effect of mango and neem extrct on four organisms causing dental caries: streptococcus mutans, streptococcus salivarius, streptococcus mitis and streptococcus sanguis: An in vivo study. Indian J Dent Res 2007; 18(4): 148-151.

11. Kanwal, Q., Hussain, I., Siddiqui, H, L., Javaid, A. Antifungal activity of flavonoids isolated from mango (Mangifera indica 1) leaves. Natural Product Research. 2010; 24(20): 1907-1914.

12. Gupta, P., Agarwal, N., Manjunath, B. C., Bhalla, A. Evaluating the anti-plaque efficacy of meswak containing dentifrices: a triple blind controlled trial. J Pharm Bioallied Sci. 2012; 4(4): 282-285.

13. Kumar, D. N., Sidhu, P. M. The antimicrobial activity of azardirachta indica, glycyrrhiza glabra, cinnamum 
zeylanicum, Syzygium aromaticum, Accacia nilotica on streptococcus mutans and enterococcus faecalis - An in vitro study. Endontology. 2011; 1(23): 101-104.

14. Kumar, M. K. P., Priya, N. K., Madhushankari, G. S. Anti-cariogenic efficacy of herbal and conventional toothpastes - a comparative in-vitro study. Journal of International Oral Health. Mar-Apr 2013; 5(2):8-13.

15. Takarada, K., Kimizuka, R., Takahashi, N., Honma, K., Okuda, K., Kato, T. A comparison of the antibacterial efficacies of essential oils against oral pathogens. Oral Microbiol Immunol. 2004: 19; 61-64.

16. Ahmad, I., Mehmood, Z., Mohammad, F. Screening of some Indian medicinal plants for their antimicrobial properties. J Ethnopharmacol. 1998 Sep; 62(2): 183-193.

17. Firas, A., Bayati, A., Khudir, D., Sulaiman. In vitro antimicrobial activity of Salvadora persica L. extracts against some isolated oral pathogenssss in Iraq, Turk J Biol. 2008; 32: 57-62.

18. Cowan, M. M. Plant products as antimicrobial agent. Clin. Microbiol. Rev. 1999; 12(4): 564.

19. Buzalaf, M. A., Pessan, J. P., Honório, H. M., Ten Cate, J. M. Mechanisms of action of fluoride for caries control. Monogr Oral Sci. 2011; 22: 97-114.

20. Perlich, M. A., Bacca, L. A., Bollmer, B. W., Lanzalaco, A. C., McClanahan, S. F., Sewak, L. K. The clinical effect of a stabilized stannous fluoride dentifrice on plaque formation, gingivitis and gingival bleeding: a six-month study. The Journal of Clinical Dentistry. 1995; 6: 54-58.

21. Severi, J. A., Lima, Z. P., Kushima, H., Brito, A. R. M. S., Santos, L.C., Vilegas, W. Polyphenols with Antiulcerogenic Action from Aqueous Decoction of Mango Leaves (Mangifera indica L.). Molecules. 2009;14: 1098-1110.

22. Nevitt, G. A., Witter, D. H., Bowman, W. D. Topical applications of sodium fluoride and stannous fluoride. Public Health Rep. 1958; 73(9): 847-850.

23. Sumanth, G., Beena, G., Bhongade, L. Oral health status of young adults using indigenous oral hygiene methods. Stomatologica India. 1992; 5: 17-23. 